\title{
Diagnóstico do Glaucoma em Imagens de Retinografia usando Variantes de Padrões Locais Binários
}

\author{
Mayara G. Silva ${ }^{1}$, Alexandre C. P. Pessoa ${ }^{1}$, João D. S. de Almeida ${ }^{1}$, \\ Geraldo Braz Junior ${ }^{1}$, Anselmo Cardoso de Paiva ${ }^{1}$ \\ ${ }^{1}$ Núcleo de Computação Aplicada (NCA), Universidade Federal do Maranhão (UFMA) \\ Caixa Postal 65.085-580 - São Luís - MA - Brazil \\ mayara@nca.ufma.br, alexandre.pessoa0707@gmail.com, jdalyson@nca.ufma.br, geraldo@nca.ufma.br, \\ paiva@nca.ufma.br
}

\begin{abstract}
Glaucoma is an optical disease that degrades the optic nerve until total loss of the field of view. The symptoms only appear when the disease is in an advanced and irreversible stage, thus, making the early diagnosis of this pathology necessary. The main objective of this work is to present a computational method that uses texture descriptors to detect glaucoma automatically in retinographies (eye fundus images). To describe the image texture, it was utilized the LBP, LQP, CS-LBP and CLBP. For image classification, SVM was utilized. The proposed method is organized in four steps: (1) pre-processing, (2) spatial decomposition, (3) feature extraction, and (4) pattern recognition. This method showed promising results of $90.70 \%$ of accuracy.
\end{abstract}

Resumo. O glaucoma é uma doença óptica que degrada o nervo óptico até a perda total do campo visual. Os sintomas só aparecem quando a doença está em estágio avançado e é irreversível, sendo assim é necessário o diagnóstico precoce de tal patologia. O principal objetivo deste trabalho é apresentar um método computacional utilizando descritores de textura para detectar o glaucoma automaticamente em retinografias (imagem de fundo de olho). Para descrever a textura foram utilizados os descritores $L B P, L Q P, C S-L B P$ e CLBP. Para a classificação das imagens foi utilizada a SVM. O método proposto está organizado em quatro etapas: (1) pré-processamento, (2) decomposição espacial, (3) extração de características e (4) reconhecimento de padrões. O método apresentou resultados promissores de 90,70\% de acurácia.

\section{Introdução}

A Pesquisa Nacional de Saúde (PNS) de 2013 revelou que dentre as deficiências físicas pesquisadas no Brasil, a com mais representatividade na população foi a visual, registrando maior proporção estimada para as pessoas que a adquiriram por doença ou acidente [Cavararo 2015]. O glaucoma é uma doença ocular e inicialmente assintomática, que causa dano ao nervo óptico ocasionando a perda irreversível do campo visual e cujo maior fator de risco é o aumento da pressão intraocular [Taleb et al. 2012]. Por ser assintomático os sintomas só aparecem quando a doença está em estágio avançado. Consequentemente, faz-se necessário o diagnóstico precoce de tal patologia.

O glaucoma geralmente tende a ser hereditário, sendo relacionado a pressão intraocular (pressão dentro do olho). Normalmente pessoas desse grupo de risco fazem 
acompanhamento da pressão intraocular, mas o diagnóstico é feito por outros tipos de exames, sendo a retinografia um dos principais. A retinografia é uma forma muito comum de se diagnosticar algumas doenças oculares, na qual utiliza-se imagem do fundo de olho para analisar o nervo óptico [Dorion 2002].

O diagnóstico do glaucoma através de imagens requer uma análise e interpretação de um especialista na área. Ao longo dos anos pesquisas na área de diagnóstico assistido por computador (CAD) vem crescendo, onde ocorre a análise de imagens computadorizadas a fim de melhorar a interpretação de exames com imagens médicas fornecendo uma padronização nas análises e uma segunda opinião para o especialista [Giger and Suzuki 2008]. As principais formas encontradas de detecção para diagnosticar o glaucoma são: por segmentação da região do disco óptico e da cavidade; e extração de características com técnicas de classificação. Na detecção por segmentação é calculado o CDR (cup-to-disc ratio), que é a razão do DO (disco óptico) com a CDO (escavação do disco óptico) e, segundo [Spaeth 1981], se o valor gerado for maior ou igual a 0,65 normalmente indica que a pessoa tem glaucoma. Um problema desse método é encontrar as regiões de interesse (ROI). Essa abordagem, por utilizar algoritmos de segmentação, pode apresentar problemas de localização para determinação de valores de limiar ou demarcação que levam a erros no diagnóstico [Sousa 2017]. Uma abordagem diferente desta é a extração de características que consideram a imagem como um todo descrevendo-as baseadas em cor, textura e/ou forma. Alguns autores [de Sousa et al. 2017, Ali et al. 2014, Araújo et al. 2017] obtiveram resultados relevantes com esta abordagem.

Este trabalho tem como objetivo avaliar a utilização de descritores de características como o LBP (Padrão Binário Local) e variantes, com a decomposição espacial juntamente com a máquina de vetores de suporte (SVM, do inglês: support vector machine) aplicados no diagnóstico do glaucoma em retinografias.

O restante deste artigo esta organizado da seguinte forma. Na Seção 2, são apresentados os trabalhos relacionados ao tema da pesquisa. Na Seção 3, são descritos os materiais e métodos utilizados neste trabalho. Os resultados obtidos, as discussões sobre esses resultados e um comparativo com outros trabalhos equivalentes ao nosso são apresentados na Seção 4. Concluindo, na Seção 5 são apresentados as conclusões da pesquisa e os trabalhos futuros em andamento ou a realizar.

\section{Trabalhos Relacionados}

Esta seção apresenta trabalhos já existentes na literatura sobre a utilização de descritores de textura em imagens médicas para diagnosticar o glaucoma em retinografias.

Ali et al. (2014) apresentam uma metodologia para a detecção automática de glaucoma, baseada em características de textura local extraídas de fotografias do fundo de olho utilizando o LBP. Utilizam padrões binários locais para capturar recursos de textura representativos da imagem inteira. Uma região local é representada por três operadores: seu pixel central (LBPC) e suas diferenças locais como dois componentes complementares, o sinal (que é o LBP clássico) e a magnitude (LBPM). A textura de imagem é descrita tanto pela distribuição de LBP quanto pela distribuição conjunta de LBPM e LBPC. As imagens foram então classificadas usando um método de vizinhança mais próxima com a estratégia de validação leave-one-out. Em um conjunto de amostras de 41 imagens de 
fundo de olho, o método atingiu taxa de sucesso de 95\%. A partir das imagens digitais de fundo de olho, Sousa (2017) propôs uma metodologia para diagnosticar o glaucoma utilizando funções geoestatísticas como: semivariograma, semimadograma, covariograma, correlograma. Inicialmente as imagens passaram por um pré-processamento na qual foram convertidas para nível de cinza e em seguidas equalizadas. Para a representação da região do disco óptico foi aplicado o Padrão Binário Local (LBP) e funções geoestatísticas para descrever os padrões de textura.

Já Araújo et al. (2017) propôs um método de diagnóstico do glaucoma também em imagens de fundo de olho utilizando índices de diversidade de Shannon e McIntosh como descritores dos padrões de textura e SVM para classificação. A aplicação dos índices de Shannon e McIntosh como descritores de textura mostrou-se eficaz alcançando como melhor resultado uma acurácia de $88,35 \%$.

Já Ramos (2016) utilizou variantes de padrões locais em imagens digitais para discriminar os padrões de massas malignas e benignas, para o diagnóstico de câncer de mama. Esta discriminação se dá através da utilização de operadores locais, das técnicas Local Binary Pattern (LBP), Local Quinary Pattern (LQP), Circular-Symmetric Local Binary Pattern (CS-LBP) e Compound Local Binary Pattern(CLBP), combinando cada uma com as abordagens Cartesian Grid, Grid X, Complete Grid, Abordagem Circular. Por fim, classificando a textura utilizando a SVM. Este estudo foi utilizado como base para o desenvolvimento desta pesquisa, onde utilizamos os mesmos descritores de características e a decomposição cartesian grid, que complementamos com pré-processamento das imagens.

\section{Materiais e Métodos}

Esta seção apresenta a base de imagens e as etapas do método proposto que está organizado em quatro etapas: (1) pré-processamento (Seção 3.2), (2) decomposição espacial(Seção 3.3), (3) extração de características (Seção 3.4) e (4) reconhecimento de padrões (Seção 3.5). A Figura 1 apresenta as etapas do método proposto.

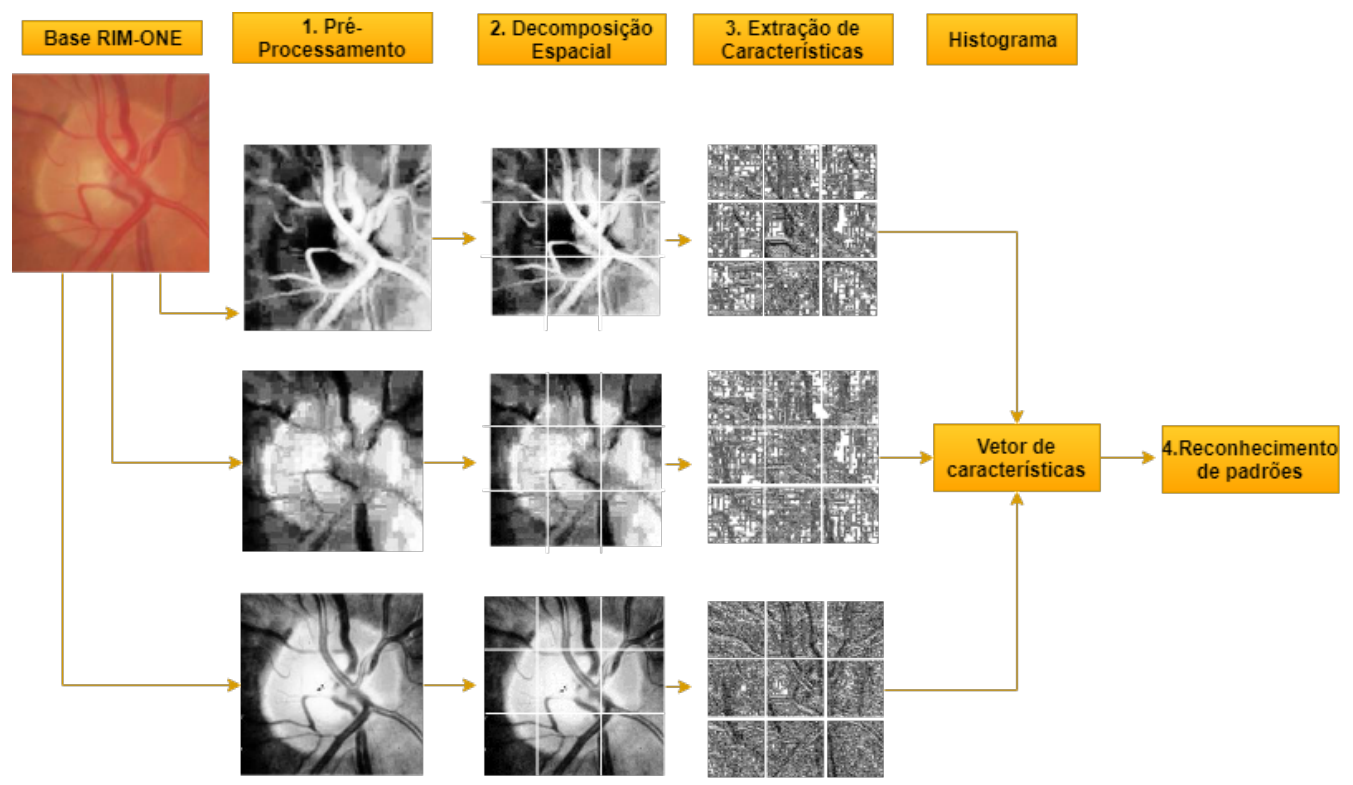

Figura 1. Etapas do método proposto. 


\subsection{Base de Imagens}

Nesta pesquisa utilizou-se a base de imagens pública RIM-ONE [Trucco et al. 2013], que é disponibilizada gratuitamente e é formada por três versões diferente. Neste trabalho foi utilizada a versão 2, que possui 455 imagens no formato RGB (Red, Green, Blue) e são separadas em duas classes: Normal (255) e Glaucoma and suspicious (200). Essas imagens foram adquiridas em três hospitais: Hospital Universitário de Canarias, Hospital Clínico San Carlos e Hospital Universitário Miguel Servet. A Figura 2 apresenta uma imagem de um indivíduo da classe Normal (Figura 2A) e de um paciente da classe Glaucoma and suspicious (Figura 2B).
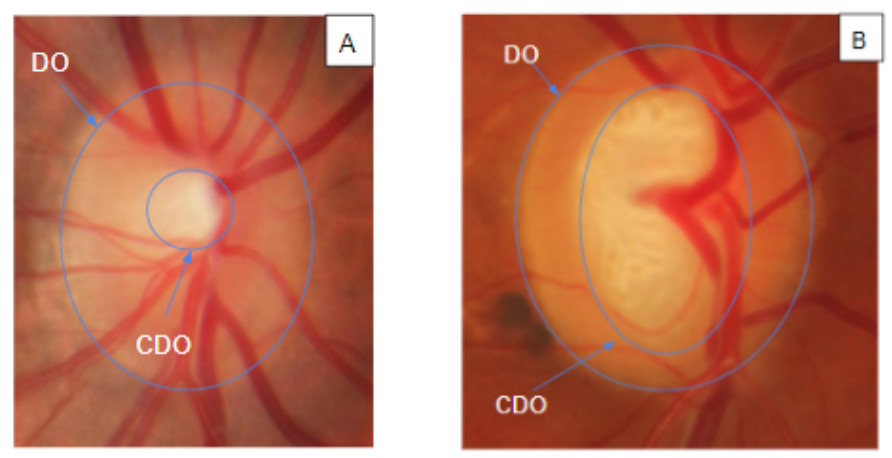

Figura 2. (A) Normal, (B) Glaucoma and suspicious.

\subsection{Pré-Processamento}

O pré-processamento teve como objetivo amenizar as diferenças de luminosidade e evidenciar características da região do disco óptico e da cavidade do disco óptico. A preparação das imagens para a extração de características foi dividida em três fases, inicialmente para diminuir o custo computacional as imagens foram redimensionadas e então a partir da imagem original (RGB) foram separados os canais de cores $R$ (vermelho), $G$ (verde), e B (azul). A Figura 3 (B) mostra o canal R em nível de cinza após a separação dos canais.

Em seguida foram aplicadas as equações de transformação de cores opostas [Hering 1880] nos três canais. As cores opostas são, vermelho versus verde, verde versus vermelho e azul versus amarelo. Segundo Sande, Gevers e Snoek (2010) os três canais opostos são calculados conforme Equação 1:

$$
R_{g}=\frac{(R-G)}{\sqrt{2}}, G_{r}=\frac{((R+G)-2 B)}{\sqrt{6}}, B_{y}=\frac{(R+G+B)}{\sqrt{3}} .
$$

A transformação em cores opostas resulta em três novas imagens. Em seguida as imagens são equalizadas [Gonzalez et al. 2004]. Na Figura 2 (C) tem-se um exemplo de uma imagem resultante após a técnica de cores opostas, a vermelho-verde. Já na Figura 2 (D) apresenta-se a imagem resultante da equalização do histograma.

A equalização de histograma é uma técnica que objetiva redistribuir os valores de tons de cinza dos pixels em uma imagem, de modo a obter um histograma uniforme, no qual o número (percentual) de pixels de qualquer nível de cinza é praticamente o mesmo 
[Marques Filho and Neto 1999]. A equalização tem papel fundamental na melhoria do contraste das imagens realçando suas características.
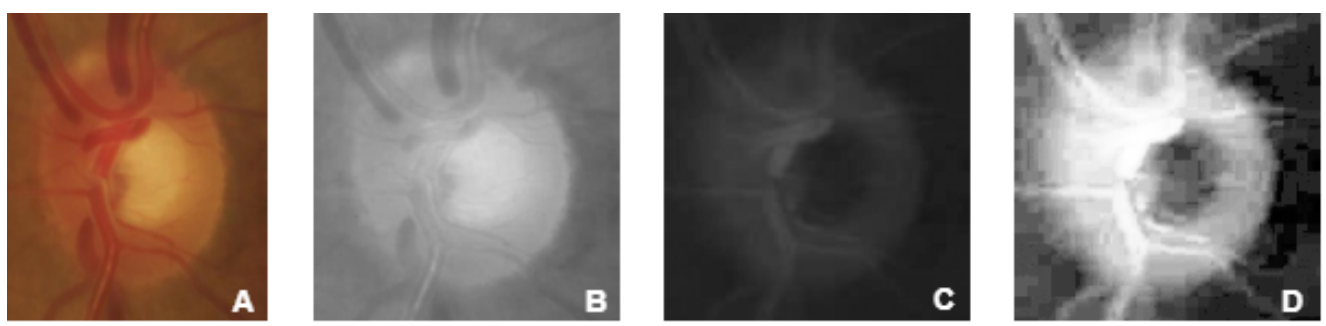

Figura 3. Pré-Processamento. (A) imagem em RGB; (B) canal R (vermelho) em nível de cinza; (C) cor oposta (vermelho-verde); (D) imagem equalizada.

\subsection{Decomposição Espacial}

Nesta etapa aplica-se na imagem uma técnica de representação visando analisar a textura localmente. Desta forma, foi utilizada a decomposição espacial Cartesian Grid [Ahmed et al. 2011].

A Cartesian Grid divide a imagem em sub-regiões como uma grade e é dada por uma função parametrizada de $M$ e $N$, onde $M$ é o número de divisões horizontais e $N$ é o número de divisões verticais [Ahmed et al. 2011]. Neste trabalho, através de testes empíricos, foi determinada a configuração do Grid na proporção 3x3. A Figura 4 representa uma das imagens com grid $3 \times 3$.

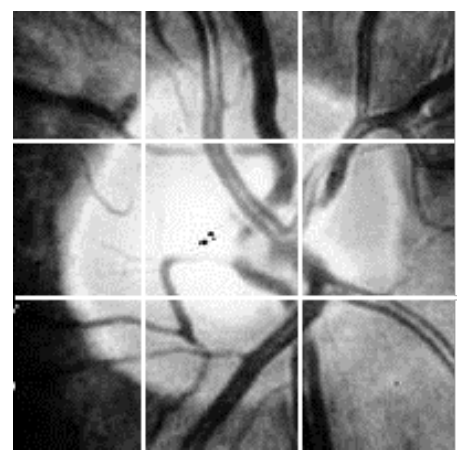

Figura 4. Exemplo de Cartesian Grid 3x3.

\subsection{Extração de Características}

$\mathrm{Na}$ etapa de extração de características foi utilizado o LBP (Local Binary Pattern) [Ojala et al. 1996] e alguns variantes como, o LQP (Local Quinary Pattern) [Nanni et al. 2012], o CS-LBP (Center-Symmetric Local Binary Pattern) [Heikkilä et al. 2006] e o CLBP (Compound Local Binary Pattern) [Ahmed et al. 2011].O LBP é um descritor de textura simples e tem sido amplamente utilizado em várias aplicações obtendo resultados relevantes [Ahonen et al. 2006]. O LBP é um operador de textura invariante aos níveis de cinza e rotação, baseado em padrões locais binários [Ramos Neto 2016]. A Equação 2 descreve o cálculo do LBP: 


$$
L B P\left(x_{c}, y_{c}\right)=\sum_{n=0}^{n-1} S\left(i_{n}-i_{c}\right) 2^{n}
$$

Onde $n$ é a quantidade de vizinhos do pixel central $\left(x_{c}, y_{c}\right), i_{c}$ é o valor de nível de cinza do pixel central $\left(x_{c}, y_{c}\right), i_{n}$ é o valor de nível de cinza de cada pixel vizinho e $\mathrm{S}(\mathrm{x})$ é uma função que devolve 1 se $x>=0 e 0$, caso contrário. A técnica LBP foi aplicada em sua forma padrão [Ojala et al. 1996], onde o cálculo é feito com 8 vizinhos e Raio 1. Foram estudados, também, os variantes LQP, CS-LBP e CLBP.

O LQP (Local Quinary Pattern) foi proposto, inicialmente, no trabalho [Nanni et al. 2012] a fim de reduzir a sensibilidade de ruídos em regiões de imagens aproximadamente uniformes, sendo um descritor relevante para a pesquisa, pois a ROI das imagens utilizadas são aproximadamente uniformes. O LQP foi aplicado em janelas $3 \times 3$, utilizando limiares $\tau_{1}$ e $\tau_{2}$, encontrados empiricamente com valores de 1 e 10 respectivamente.

O CS-LBP também foi aplicado em janelas 3x3, utilizando limiar 10 para o cálculo do CS-LBP. O CS-LBP é um descritor que visa reduzir o tamanho dos histogramas gerados pelo operador do LBP, comparando pares de pixels simétricos em relação ao centro, produzindo assim histogramas mais compactos [Heikkilä et al. 2006].

O CLBP também foi calculado em janela $3 \times 3$ e para calcular o threshold foi utilizada a média dos módulos das diferenças entre o centro e os valores de nível de cinza vizinhos. O CLBP [Ahmed et al. 2011] é uma variação do operador LBP. Ao contrário do LBP que utiliza somente a informação gerada pelos sinais das diferenças entre o pixel central e os vizinhos, o CLBP também utiliza a magnitude dessa diferença, que é uma importante informação sobre a textura, mostrando bons resultados e até melhores quando comparado ao LBP [Ramos Neto 2016].

Em seguida, para cada abordagem foram gerados histogramas parciais (aplicado em cada sub-região resultante da abordagem de decomposição utilizada), que foram normalizados e concatenados de forma que no reconhecimento de padrões as características de cada sub-região sejam comparadas somente com seu correspondente nas outras imagens, por fim formando o vetor de características.

\subsection{Reconhecimento de Padrões}

Nesta etapa o objetivo foi classificar cada imagem em glaucomatosa ou saudável, utilizando o reconhecimento de padrões. Os resultados foram avaliados analisando-se o desempenho de cada um dos métodos utilizados, decomposição/descritor, classificando as imagens em saudáveis e glaucomatosas por meio do uso do SVM [Vapnik 1999]. O SVM mapeia os dados para um espaço e em seguida encontra um hiperplano de separação linear com margem para dividir em classes diferentes [Rivera et al. 2012]. Dado o conjunto de treinamento $\left(x_{i}, y_{i}\right)$, o objetivo da classificação é estimar uma função que separe corretamente os exemplos de teste em classes distintas [Ahmed et al. 2011]. O SVM foi utilizado com Função de Base Radial (RBF).

Na realização dos experimentos, foram utilizadas as 455 imagens da base (Seção 3.1). A classificação foi realizada seguindo as classes atribuídas pelo especialista na base de imagens. Para os experimentos, foi utilizado o método de aprendizagem de máquina 
com divisão proporcional da base para treino e teste, utilizando as respectivas proporções, de 50/50, 60/40, 70/30 e 80/20. Os resultados foram avaliados utilizando a abordagem de validação cruzada.

Em posse dos resultados gerados, a configuração que obteve melhor resultado foi submetida a seleção de características. A seleção foi feita pelo algoritmo PSO [Eberhart and Kennedy 1995], em seguida as instâncias com as características selecionadas foram submetidas ao classificador SVM. A capacidade de generalização do classificador foi avaliada através do método de validação cruzada $\mathrm{k}$-fold (com $k=10)$.

\subsection{Validação dos Resultados}

A validação dos resultados tem como objetivo avaliar o desempenho e promover a validação dos resultados obtidos. Neste trabalho foram utilizadas as estatísticas: Acurácia, Sensibilidade e Especificidade [Chimieski and Fagundes 2013].

Segundo Sousa (2017) para análise de amostra de determinada doença com caso positivo e negativo, os resultados dos testes de classificação dos casos analisados podem ser divididos em quatro grupos:

- VP : Verdadeiro Positivo - o teste é positivo e o paciente tem glaucoma.

- FP : Falso Positivo - o teste é positivo, mas o paciente não tem glaucoma.

- VN : Verdadeiro Negativo - o teste é negativo e o paciente não tem glaucoma.

- FN : Falso Negativo - o teste é negativo, mas o paciente tem glaucoma.

A sensibilidade (SEN) é o número de imagens corretamente classificadas como glaucomatosas.

$$
S E N=\frac{V P}{V P+F N}
$$

A especificidade (ESP) é o número de imagens classificadas corretamente como saudáveis.

$$
E S P=\frac{V N}{V N+F P}
$$

A acurácia (AC) é a proporção total dos resultados corretos. Definida como a razão entre o número de casos na amostra em estudo que foram classicados corretamente e o número total de amostras.

$$
A C U=\frac{V P+V N}{V P+F P+F N+V N}
$$

\section{Resultados e Discussões}

Nesta seção são apresentados os resultados obtidos por este estudo e sua análise. Foram utilizadas todas as imagens da base, cada imagem pré-processada gerou 3 imagens e com a aplicação da decomposição espacial, cada imagem gerou 9, totalizando vinte e sete imagens. Em cada uma dessas imagens foram aplicados os descritores de características, cada 
descritor gerando uma quantidade de imagens: LBP (1), CS-LBP (1), LQP (4) e CLBP (2). Foram realizadas para cada proporção treino/teste cinco repetições e os parâmetros estimados, usando validação cruzada. A seguir, apresentaremos os resultados para cada uma das abordagens de extração de características utilizadas durante o desenvolvimento deste trabalho.

Na Tabela 1 pode-se observar que para o descritor LBP o melhor resultado foi obtido na proporção 80/20 com sensibilidade média de 86,50\% e acurácia média de 87,25\%. Porém a especificidade foi melhor na proporção 50/50, mas não houve uma diferença significativa.

Tabela 1. Teste do Cartesian grid $3 \times 3$ com LBP

\begin{tabular}{c|l|l|l}
\hline Treino/Teste (\%) & Acurácia (\%) & Sensibilidade (\%) & Especificidade (\%) \\
\hline $50 / 50$ & $84,29 \pm 0,01$ & $78,20 \pm 0,04$ & $89,06 \pm 0,01$ \\
$60 / 40$ & $84,17 \pm 0,03$ & $78,50 \pm 0,06$ & $88,62 \pm 0,02$ \\
$70 / 30$ & $83,79 \pm 0,02$ & $79,66 \pm 0,05$ & $87,01 \pm 0,01$ \\
$\mathbf{8 0 / 2 0}$ & $\mathbf{8 7 , 2 5} \pm \mathbf{0 , 0 1}$ & $\mathbf{8 6 , 5 0} \pm \mathbf{0 , 0 3}$ & $\mathbf{8 7 , 8 4} \pm \mathbf{0 , 0 1}$ \\
\hline
\end{tabular}

Para o descritor LQP, os testes apresentados na Tabela 2 demostraram que foram obtidos melhores resultados na proporção 50/50, com especificidade média de 89,21\% e acurácia média de $84,73 \%$, porém a sensibilidade foi melhor na proporção 80/20 com média de $86,50 \%$. Na Tabela 3 estão os resultados com o descritor CS-LBP que, assim como o LQP obteve melhor resultado na proporção 50/50 com sensibilidade média de $62,40 \%$, especificidade média de 75,93\% e acurácia média de $70 \%$.

Tabela 2. Teste do Cartesian grid $3 \times 3$ com LQP.

\begin{tabular}{c|l|l|l}
\hline Treino/Teste (\%) & Acurácia (\%) & Sensibilidade (\%) & Especificidade (\%) \\
\hline $\mathbf{5 0 / 5 0}$ & $\mathbf{8 4 , 7 3} \pm \mathbf{0 , 0 1}$ & $\mathbf{7 9 , 0 0} \pm \mathbf{0 , 0 4}$ & $\mathbf{8 9 , 2 1} \pm \mathbf{0 , 0 2}$ \\
$60 / 40$ & $83,29 \pm 0,03$ & $78,75 \pm 0,07$ & $86,86 \pm 0,01$ \\
$70 / 30$ & $82,48 \pm 0,02$ & $79,66 \pm 0,06$ & $84,67 \pm 0,01$ \\
$80 / 20$ & $81,00 \pm 0,04$ & $86,50 \pm 0,10$ & $85,88 \pm 0,03$ \\
\hline
\end{tabular}

Tabela 3. Teste do Cartesian grid $3 \times 3$ com CS-LBP

\begin{tabular}{c|l|l|l}
\hline Treino/Teste $(\%)$ & Acurácia $(\%)$ & Sensibilidade $(\%)$ & Especificidade (\%) \\
\hline $\mathbf{5 0 / 5 0}$ & $\mathbf{7 0 , 0 0} \pm \mathbf{0 , 0 4}$ & $\mathbf{6 2 , 4 0} \pm \mathbf{0 , 0 6}$ & $\mathbf{7 5 , 9 3} \pm \mathbf{0 , 1 0}$ \\
$60 / 40$ & $68,79 \pm 0,04$ & $61,50 \pm 0,11$ & $74,50 \pm 0,08$ \\
$70 / 30$ & $67,00 \pm 0,03$ & $61,00 \pm 0,02$ & $71,68 \pm 0,07$ \\
$80 / 20$ & $67,03 \pm 0,08$ & $56,00 \pm 0,06$ & $75,68 \pm 0,11$ \\
\hline
\end{tabular}

Os testes com o descritor CLBP (Tabela 4) demostraram resultados de acurácia e especificidade melhores que os demais, com uma acurácia média de $89,45 \%$ e especificidade média de 92,54\% na proporção treino/teste 80/20, seguido do LBP que obteve uma acurácia de 87,25\%. Analisando esses resultados, percebe-se que o LBP e CLBP na 
proporção de treino/teste 80/20 apresentaram melhores índices, já o LQP e CS-LBP tiveram melhores resultados com 50/50. Observa-se também que os resultados apresentam valores de especificidade sempre maiores que os de sensibilidade, indicando que o método se mostra mais eficiente na detecção de casos saudáveis. Os resultados demonstram, ainda, que através do baixo desvio padrão resultante entre as proporções de treino/teste, existe um poder de generalização do método empregado, embora novas investigações devam ser realizadas a fim de verificar a complexidade do modelo gerado.

Tabela 4. Teste do Cartesian grid $3 \times 3$ com CLBP

\begin{tabular}{c|l|l|l}
\hline Treino/Teste (\%) & Acurácia (\%) & Sensibilidade (\%) & Especificidade (\%) \\
\hline $50 / 50$ & $85,87 \pm 0,02$ & $81,20 \pm 0,05$ & $89,53 \pm 0,01$ \\
$60 / 40$ & $85,16 \pm 0,01$ & $79,25 \pm 0,04$ & $89,80 \pm 0,02$ \\
$70 / 30$ & $86,42 \pm 0,01$ & $82,33 \pm 0,05$ & $89,61 \pm 0,01$ \\
$\mathbf{8 0 / 2 0}$ & $\mathbf{8 9 , 4 5} \pm \mathbf{0 , 0 2}$ & $\mathbf{8 5 , 5 0} \pm \mathbf{0 , 0 5}$ & $\mathbf{9 2 , 5 4} \pm \mathbf{0 , 0 1}$ \\
\hline
\end{tabular}

A Tabela 5 apresenta os resultados com a seleção de características. Pode-se observar que o resultado do descritor CLBP após a seleção de características aumentou relativamente a acurácia, de $89,45 \%$ para $90,75 \%$.

Tabela 5. Comparação dos resultados do CLBP após a seleção de características

\begin{tabular}{c|c|c|c|}
\hline Qtd. Características & Acurácia (\%) & Sensibilidade (\%) & Especificidade (\%) \\
\hline 13824 & 89,40 & 85,50 & 92,50 \\
2822 & 90,70 & 90,80 & 90,80 \\
\hline
\end{tabular}

Diante dos resultados obtidos, a Tabela 6 mostra uma breve comparação dos resultados encontrados neste trabalho com alguns trabalhos relacionados. São apresentadas a metodologia utilizada, quantidade de amostras, e as métricas de validação. Este trabalho comparado aos que usam a mesma base de imagens e também o LBP, ficou com o segundo melhor resultado em todas as estatísticas, com resultado de acurácia superior e sensibilidade consideravelmente melhor ao do trabalho de Araujo et al. (2017) e resultado da acurácia um pouco inferior a Sousa (2017), porém a especificidade foi ligeiramente maior. A maioria dos trabalhos relacionados utilizou a mesma base e quantidade de amostras, exceto o trabalho de Ali et al. (2014) que possui o melhor resultado entre todos os trabalhos. Esse, porém, utiliza uma quantidade consideravelmente menor de imagens, significando uma baixa variabilidade de casos, acarretando assim, em melhores resultados.

Tabela 6. Comparação com os trabalhos relacionados

\begin{tabular}{l|l|c|c|c|c}
\hline Autor & Características & Imagens & ACU. & SEN. & ESP. \\
\hline Sousa (2017) & Funções Geoestatísticas & 455 & 91,20 & 95,00 & 88,20 \\
Ali et al. (2014) & LBP & 41 & 95,00 & 96,00 & 92,00 \\
Araújo et al. (2017) & Índices de diversidade & 455 & 88,30 & 84,50 & 91,30 \\
Método proposto & CLBP & $\mathbf{4 5 5}$ & $\mathbf{9 0 , 7 0}$ & $\mathbf{9 0 , 8 0}$ & $\mathbf{9 0 , 8 0}$ \\
\hline
\end{tabular}




\section{Conclusão}

Para objetivo deste trabalho, foram comparadas 4 técnicas de análise de textura juntamente com a técnica de decomposição espacial cartesiam grid, visando o uso no de diagnóstico de imagens glaucomatosas. Os resultados obtidos entre o cruzamento desses métodos revelaram que o cartesian grid e o CLBP junto com SVM e o pré-processamento das cores opostas obtiveram resultados promissores apresentando um índice de 90,7\% de acurácia.

Como trabalhos futuros, a fim de ampliar e melhorar os resultados, sugere-se a realização de testes com outros métodos de aprendizagem de máquina como o Random Forest, utilizar outras estratégias de extração de características baseado em Deep Learning, testar a estratégia de seleção de instâncias realizada por Sousa (2017) e realizar teste em um volume maior de imagens.

\section{Agradecimentos}

Os autores agradecem à FAPEMA (Processo: UNIVERSAL-01082/16) e ao CNPq (Processo: 423493/2016-7) pelo apoio financeiro.

\section{Referências}

Ahmed, F., Hossain, E., Bari, A. H., and Shihavuddin, A. (2011). Compound local binary pattern (clbp) for robust facial expression recognition. In Computational Intelligence and Informatics (CINTI), 2011 IEEE 12th International Symposium on, pages 391395. IEEE.

Ahonen, T., Hadid, A., and Pietikainen, M. (2006). Face description with local binary patterns: Application to face recognition. IEEE transactions on pattern analysis and machine intelligence, 28(12):2037-2041.

Ali, M. A., Hurtut, T., Faucon, T., and Cheriet, F. (2014). Glaucoma detection based on local binary patterns in fundus photographs. In SPIE Medical Imaging, pages 903531903531.

Araújo, J. D. L., de Paiva, A. C., De Almeida, J. D., Neto, O. P. S., de Sousa, J. A., Silva, A. C., and Júnior, G. B. (2017). Diagnóstico de glaucoma em imagens de fundo de olho utilizando osíndices de diversidade de shannon e mcintosh. XVII Workshop de Informática Médica.

Cavararo, R. (2015). Pesquisa nacional de saúde 2013. instituto brasileiro de geografia e estatística-ibge. diretoria de pesquisas. coordenação de trabalho e rendimento, rio de janeiro, 2015.

Chimieski, B. F. and Fagundes, R. D. R. (2013). Association and classification data mining algorithms comparison over medical datasets. Journal of health informatics, $5(2)$.

de Sousa, J. A., de Paiva, A. C., de Almeida, J. D. S., Silva, A. C., Junior, G. B., and Gattass, M. (2017). Texture based on geostatistic for glaucoma diagnosis from fundus eye image. Multimedia Tools and Applications, pages 1-18.

Dorion, T. (2002). Manual de Exame Do Fundo de Olho. Manolen. 
Eberhart, R. and Kennedy, J. (1995). A new optimizer using particle swarm theory. In Micro Machine and Human Science, 1995. MHS'95., Proceedings of the Sixth International Symposium on, pages 39-43. IEEE.

Giger, M. L. and Suzuki, K. (2008). Computer-aided diagnosis. In Biomedical information technology, pages 359-XXII. Elsevier.

Gonzalez, R. C. E., Woods, S. L., Gonzalez, R. E. R. E. R. C., Woods, R. E., and Eddins, S. L. (2004). Digital image processing using MATLAB. Number 04; TA1637, G6.

Heikkilä, M., Pietikäinen, M., and Schmid, C. (2006). Description of interest regions with center-symmetric local binary patterns. In ICVGIP, volume 6, pages 58-69. Springer.

Hering, E. (1880). Zur Erklärung der Farbenblindheit aus der Theorie der Gegenfarben, volume 1. Tempsky.

Marques Filho, O. and Neto, H. V. (1999). Processamento digital de imagens. Brasport.

Nanni, L., Lumini, A., and Brahnam, S. (2012). Survey on lbp based texture descriptors for image classification. Expert Systems with Applications, 39(3):3634-3641.

Ojala, T., Pietikäinen, M., and Harwood, D. (1996). A comparative study of texture measures with classification based on featured distributions. Pattern recognition, 29(1):5159.

Ramos Neto, G. M. (2016). Estudo de Variantes de Padrões Locais Binários para o Diagnóstico de Câncer de Mama. Monografia (Bacharel em Ciência da Computação), UFMA (Universidade Federal do Maranhão), São Luís, Brasil.

Rivera, A. R., Castillo, J. A. R., and Chae, O. (2012). Recognition of face expressions using local principal texture pattern. In Image Processing (ICIP), 2012 19th IEEE International Conference on, pages 2609-2612. IEEE.

Sousa, J. A. d. (2017). Diagnóstico de glaucoma em retinografias utilizando funções geoestatística. Dissertação (Mestrado) - Universidade Federal do Maranhão, Curso de Pós Graduação em Ciência da Computação, São Luís, MA.

Spaeth, G. (1981). Appearances of the optic disc in glaucoma: a pathogenetic classification. In Trans Acad Ophthalmol Symposium on Glaucoma. CV Mosby, St. Louis.

Taleb, A., Faria, M. A. R. d., Ávila, M., and Mello, P. (2012). As condições de saúde ocular no brasil-2012. Conselho Brasileiro de Oftalmologia. São Paulo.

Trucco, E., Ruggeri, A., Karnowski, T., Giancardo, L., Chaum, E., Hubschman, J. P., Al-Diri, B., Cheung, C. Y., Wong, D., Abramoff, M., et al. (2013). Validating retinal fundus image analysis algorithms: Issues and a proposalvalidating retinal fundus image analysis algorithms. Investigative ophthalmology \& visual science, 54(5):3546-3559.

Vapnik, V. N. (1999). An overview of statistical learning theory. IEEE transactions on neural networks, 10(5):988-999. 